\title{
Clinical and radiographic outcomes following traumatic Grade 3 and 4 carotid artery injuries: a 10 -year retrospective analysis from a Level 1 trauma center. The Parkland Carotid and Vertebral Artery Injury Survey
}

\author{
William W. Scott, MD, ${ }^{1}$ Steven Sharp, MD, ${ }^{1}$ Stephen A. Figueroa, MD, ${ }^{2}$ \\ Alexander L. Eastman, MD, MPH, ${ }^{3}$ Charles V. Hatchette, MD, ${ }^{1}$ Christopher J. Madden, MD, ${ }^{1}$ \\ and Kim L. Rickert, MD ${ }^{1}$
} Departments of ${ }^{1}$ Neurosurgery, ${ }^{2}$ Neurocritical Care, and ${ }^{3}$ Trauma Surgery, University of Texas Southwestern Medical Center,
Dallas, Texas

\begin{abstract}
OBJECT Screening, management, and follow-up of Grade 3 and 4 blunt carotid artery injuries (BCAls) remain controversial. These high-grade BCAls were analyzed to define their natural history and establish a rational management plan based on lesion progression and cerebral infarction.

METHODS A retrospective review of a prospectively maintained database of all blunt traumatic carotid and vertebral artery injuries from August 2003 to April 2013 was performed, and Grade 3 and 4 BCAls were identified. The authors define Grade 3 injuries as stenosis of the vessel greater than 50\%, or the development of a pseudoaneurysm, and Grade 4 injuries as complete vessel occlusion. Demographic information, imaging findings, number of images obtained per individual, length of radiographic follow-up examination, radiographic outcome at end of follow-up period, treatment(s), and documentation of ischemic stroke or transient ischemic attack (TIA) were recorded.
\end{abstract}

RESULTS Fifty-three Grade 3 BCAls in 44 patients and 5 Grade 4 BCAls in 5 patients were identified and had available follow-up information. The mean follow-up duration for Grade 3 BCAls was 113 days, and the mean follow-up for Grade 4 BCAls was 78 days. Final imaging of Grade 3 BCAls showed that $53 \%$ of cases were radiographically stable, $11 \%$ had resolved, and $11 \%$ were improved, whereas $25 \%$ had radiographically worsened. In terms of treatment, $75 \%$ of patients received aspirin (ASA) alone, $5 \%$ received various medications, and $2 \%$ received no treatment. Eighteen percent of the patients in the Grade $3 \mathrm{BCAl}$ group underwent endovascular intervention, and in all of these cases, treatment with ASA was continued after the procedure. Final imaging of the Grade 4 BCAls showed that $60 \%$ remained stable (with persistent occlusion), whereas the remaining arteries improved (with recanalization of the vessel). All patients in the Grade 4 BCAI follow-up group were treated with ASA, although in 1 patient treatment was transitioned to Coumadin. There were 3 cases of cerebral infarction that appeared to be related to Grade 3 BCAls (7\% of 44 patients in the Grade 3 group), and 1 case of stroke that appeared to be related to a Grade 4 BCAl. All identified cases of stroke developed soon after hospital admission.

CONCLUSIONS Although the posttraumatic cerebral infarction rate may be overestimated, the results of this study suggest that the Grade 3 and 4 BCAls carry the highest stroke risk of the blunt cerebrovascular injuries, and those infarctions were identified on or shortly after hospital admission. Despite a 40\% recanalization rate in the Grade $4 \mathrm{BCAl}$ group and an $89 \%$ rate of persistent pseudoaneurysm in the Grade 3 BCAI group, follow-up imaging showed progressive worsening without radiographic improvement in only a small number of patients, and these findings alone did not correlate with adverse clinical outcome. Follow-up protocols may require amending; however, further prospective studies are needed to make conclusive changes as they relate to management.

http://thejns.org/doi/abs/10.3171/2014.10.JNS14875

KEY WORDS blunt cervical vascular injury; carotid artery injury; cerebral infarction; trauma

ABBREVIATIONS ASA = aspirin; BCAI = blunt carotid artery injury; BCVI = blunt cerebrovascular injury; $\mathrm{BVAI}=$ blunt vertebral artery injury; $\mathrm{CTA}=\mathrm{CT}$ angiography; $\mathrm{DSA}=$ digital subtraction angiography; MVC = motor vehicle collision; PCVAIS = Parkland Carotid and Vertebral Artery Injury Survey.

SUBMITTED April 18, 2014. ACCEPTED October 27, 2014.

INCLUDE WHEN CITING Published online December 19, 2014; DOI: 10.3171/2014.10.JNS14875.

DISCLOSURE The authors report no conflict of interest concerning the materials or methods used in this study or the findings specified in this paper. 
$\mathrm{T}$ HE management of blunt cerebrovascular injuries (BCVIs) remains controversial; however, antiplatelet agents or anticoagulant medications continue to be first-line treatments, with endovascular stenting typically reserved for symptomatic or certain higher-grade injuries. $5,7,8,14,19,28$ Although our earlier reports explored blunt vertebral artery injuries (BVAIs) and lower-grade blunt carotid artery injuries (BCAIs), this article evaluates the higher-grade BCAIs (Grade 3 and 4). Imaging, progression of injury, and outcomes of these high-grade BCAIs were analyzed to gain a better understanding of their natural history and to assist in establishing a rational management plan based on their progression and risk of cerebral infarction.

\section{Methods}

We performed a retrospective review of a prospectively maintained database of all traumatic carotid and vertebral artery injuries at our Level I trauma center (Parkland Memorial Hospital, Dallas, TX). The focus of this was on the Grade 3 and 4 BCAIs. We suspect that lower-grade injuries may exhibit a more benign behavior compared with higher-grade injuries; therefore, our aim for the Parkland Carotid and Vertebral Artery Injury Survey (PCVAIS) was to categorize and report on these injuries separately.

Screening for BCVIs at our institution occurs through a modification of the Denver Criteria. Any patient found to have cervical spine fractures involving the vertebral body or pedicle, basilar skull fractures involving the carotid canal or petrous bone, Le Fort II or III fractures, and a Glasgow Comma Scale Score $<7$ without obvious CT-documented cerebral injury, and/or near hanging/ strangulation with a ligature mark/contusion undergoes cervical CT angiography (CTA) screening to rule out a vascular injury. Although variability exists between providers and clinical scenarios, the desired radiographic follow-up examination includes a repeat head and neck CTA at 1-week, 1-month, and 3-month intervals as long as the injury remained present.

Grade 1 injuries are defined as a vessel lumen stenosis of less than $25 \%$ and Grade 2 injuries as a stenosis of the vessel lumen between $25 \%$ and $50 \%$. We define Grade 3 injuries as stenosis of the vessel greater than $50 \%$ or the development of a pseudoaneurysm, and Grade 4 injuries as vessel occlusion. ${ }^{3}$ Grade 5 injuries are complete transections of the artery. All CTA images were assessed by a staff member from our department of neuroradiology. A staff neurosurgeon confirmed all injuries and decided on the desired treatment and follow-up examination. Only blunt injuries were evaluated. Patients with vascular injuries due to penetrating trauma were not included in this analysis.

Data, including age, sex, mechanism of injury, presence or absence of a cervical fracture, and other traumatic injuries within the proximity of the neck (e.g., occipital condyle fracture, first rib fracture, etc.), were recorded. Imaging studies (e.g., CTA, digital subtraction angiography [DSA]) were reviewed, and we documented the number of images acquired for each individual, the length of time between imaging sessions, and radiographic outcomes at the end of the follow-up period. Treatment(s) was recorded. The pres- ence of cerebral infarction was confirmed by clinical presentation and neuroimaging.

\section{Results}

From August 2003 to April 2013, there were 537 recorded cases of blunt cervical vascular injuries, which included 241 BCAIs (45\%) and 296 BVAIs (55\%). Of these cases, there were 68 Grade 3 BCAIs in 57 patients. This comprised $13 \%$ of all blunt cervical vascular injuries and $28 \%$ of all BVAIs. Of these 57 patients, 44 patients with 53 Grade 3 BCAIs were available for follow-up examination, and their data were analyzed further in this study. There were 21 left-sided, 14 right-sided, and 9 bilateral Grade 3 BCAIs. There were 23 male and 21 female patients. The mean age of the patients with Grade 3 BCAIs was 35 years (range 16-66 years). There were 8 patients with Grade 4 BCAIs; 5 of these patients were available for follow-up. There were 3 left-sided and 2 right-sided Grade 4 BCAIs in 4 male patients and 1 female, whose average age was 21 years (range 18-24 years). Table 1 provides a summary of additional demographic and injury-related information for the Grade 4 BCAIs.

Fourteen patients were excluded from the Grade 3 BCAI group. Six of the excluded individuals, with a mean age of 40 years (range 21-85 years), were either dead on arrival $(n=2)$ or died shortly after admission $(n=4)$. In the 4 who died shortly after admission, the cause was severe traumatic brain injury or other type of traumatic injury. Eight excluded patients were lost to follow-up but had been hospitalized for a mean of 13 days (range 1-60 days). The 3 patients excluded from the Grade 4 BCAI group were older on average, with a mean age of 64 years (range 35-82 years). All 3 individuals presented with a Glasgow Coma Scale score of 3-4, and all were pronounced dead either on arrival or shortly after hospital admission (Table 1).

The mechanism of injury for the 44 patients with Grade 3 BCAIs was motor vehicle collision (MVC) in 29 patients (66\%), motor vehicle-pedestrian collision in 6 patients $(14 \%)$, motorcycle collision in 2 patients, fall in 3 patients, assault in 3 patients, and an all-terrain vehicle accident in 1 patient. In the 5 Grade 4 BCAIs that were followed, the mechanism of injury was MVC in 3 cases, motor vehiclepedestrian collision in 1 case, and a fall in 1 case.

Of the 44 patients with Grade 3 BCAIs, 17 (39\%) had an isolated, single-sided Grade 3 BCAI, whereas 27 patients $(61 \%)$ presented with multiple cervical vascular injuries (an additional ipsilateral and/or contralateral BCAI and/or BVAI). The distribution of other vascular injuries is shown in Table 2. Cervical fractures were present in 13 patients (30\%). Skull base fractures were present in 12 patients (27\%), with only 4 of these patients demonstrating involvement of the carotid canal. First rib fractures were present in 7 patients $(16 \%)$, ipsilateral occipital condyle fractures were present in 5 patients (11\%), and ipsilateral mandibular fractures were present in 2 patients $(5 \%)$, with 1 of these fractures in close proximity to the vascular lesion. Although only $20 \%$ of the Grade 4 BCAI follow-up group had cervical fractures, $80 \%$ harbored skull base fractures, with only 1 patient demonstrating involvement of the carotid canal. In 1 case, there was an ipsilateral mandible fracture present in proximity to the vascular lesion. 
TABLE 1. Demographic and outcome data for Grade 4 BCAls

\begin{tabular}{|c|c|c|c|c|c|c|c|c|}
\hline $\begin{array}{c}\text { Age (yrs) } \\
\text { Sex }\end{array}$ & $\begin{array}{l}\text { Injury } \\
\text { Mech }\end{array}$ & Fractures & $\begin{array}{l}\text { BCAI } \\
\text { Side }\end{array}$ & Other Injury & Imaging & Treatment & $\begin{array}{c}\text { FU } \\
\text { (wks) }\end{array}$ & Outcome \\
\hline $76, \mathrm{~F}$ & MVC & C-7, clinoid & $\mathrm{Lt}$ & $\begin{array}{l}\text { Lt Gr } 2 \text { BVAl, Rt Gr } \\
2 \text { BVAl }\end{array}$ & $\begin{array}{l}\text { CTA } \times 1 \\
\quad \text { DSA } \times 1\end{array}$ & Heparin & 1 & Pronounced dead shortly after admission \\
\hline $35, M$ & MPC & C-7, It 1st rib & Rt & $\begin{array}{l}\text { Lt Gr } 2 \text { BCAl, Lt Gr } \\
\text { 4 BVAl }\end{array}$ & $\mathrm{CTA} \times 1$ & None & 0 & Arrived dead \\
\hline $24, M^{*}$ & MVC & C-3, skull base & Rt & None & $\begin{array}{l}\mathrm{CTA} \times 3 \\
\quad \mathrm{DSA} \times 1\end{array}$ & $\begin{array}{l}\text { ASA } \rightarrow \\
\quad \text { Coumadin }\end{array}$ & 5 & Stable occlusion $†$ \\
\hline $82, \mathrm{M}$ & MVC & C-3, C-4, C-6, C-7 & $\mathrm{Lt}$ & Lt Gr 4 BVAl & $\mathrm{CTA} \times 1$ & None & 0 & Pronounced dead shortly after admission \\
\hline $24, M^{*}$ & MVC & C-4, rt mandible & Rt & None & $\mathrm{CTA} \times 2$ & ASA & 2 & Stable occlusion \\
\hline $21, M^{*}$ & Fall & C-2, skull base & $\mathrm{Lt}$ & None & $\mathrm{CTA} \times 2$ & ASA & 1.5 & Recanalized on 2nd scan \\
\hline $20, F^{*}$ & MVC & C-2, C-3, skull base & Lt & Lt Gr 3 BCAI & $\mathrm{CTA} \times 5$ & ASA & 22 & Stable occlusion \\
\hline $18, M^{*}$ & MPC & C-2, clinoid & $\mathrm{Lt}$ & Rt Gr 1 BCAl & $\mathrm{CTA} \times 5$ & ASA & 25 & Recanalized on 2nd scan \\
\hline
\end{tabular}

$\mathrm{FU}=$ follow-up; $\mathrm{Gr}=$ Grade; Mech = mechanism.

* Noted to have stable neurological exam at last follow-up.

† Case involved with clinically asymptomatic BCl-related cerebral infarction.

In the Grade 3 BCAI group, there were 33 patients who were evaluated solely with CTA: 6 patients received 2 scans, 13 patients received 3 scans, 4 patients received 4 scans, 7 patients received 5 scans, 2 patients received 6 scans, and 1 patient received 7 scans. The remaining patients underwent a combination of CTA and DSA. Eight of those patients also received endovascular treatment: stenting in 7 and coil embolization in 1. DSA was more likely to be performed early in our experience (between 2003 and 2007); its use decreased thereafter (once in 2009, once in 2010, and once in 2012). Likewise, endovascular intervention was performed early in our experience, with the majority of interventions occurring between 2003 and 2005. Stenting was performed for the following reasons: increased size of the pseudoaneurysm seen on the first follow-up scan in 5 patients $(71 \%)$ and progressive pseudoaneurysm-related stenosis in 1 patient; in 1 patient, endovascular stenting of the affected vessel was performed soon after the diagnosis of the Grade 3 BCAI and before follow-up imaging. The one case in which coil embolization was performed occurred in 2005, soon after the patient was diagnosed with Grade 3 BCAI and before follow-up imaging.

On final imaging of the 53 Grade 3 BCAIs, 6 showed complete resolution (11\%), 6 showed radiographic improvement $(11 \% ; 3$ showed a reduction in grade, and 3 showed a decrease in the size of the pseudoaneurysm), and 28 were radiographically stable (53\%); however, in 8 of these cases, the stability seemed to be the result of an endovascular pro-

TABLE 2. Distribution of BVAls and BCAls in relation to Grade 3 BCAls

\begin{tabular}{cccccc}
\hline & \multicolumn{2}{c}{ Vertebral Injury } & & \multicolumn{2}{c}{ Carotid Injury } \\
\cline { 2 - 3 } \cline { 5 - 6 } Grade & Ipsilat & Contralat & & Ipsilat & Contralat \\
\hline 1 & 6 & 7 & & 5 & 4 \\
\hline 2 & 2 & 2 & & 0 & 4 \\
\hline 3 & 3 & 0 & & 0 & 7 \\
\hline 4 & 1 & 1 & & 0 & 1 \\
\hline
\end{tabular}

cedure. Twelve of the injuries exhibited radiographic worsening, as defined by increasing pseudoaneurysm size, and in 5 of these cases, endovascular stenting was performed as result of this worsening. In one patient, the injured artery worsened by a grade (progression to a Grade 4 BCAI), which was noted on the first follow-up CTA image. In this latter case, the Grade 4 injury improved subsequently to a Grade $3 \mathrm{BCAI}$ at the third follow-up imaging session and the lesion remained stable thereafter. Table 3 shows the status of the Grade 3 injuries at 7-10 days postoperatively and at final imaging follow-up. The average duration of follow-up in the Grade 3 BCAI group was 113 days (range 7-960 days). As depicted in Table 1, final imaging of the Grade 4 BCAI follow-up group showed that $60 \%$ of the lesions were stable (with persistent occlusion), whereas the remaining cases improved in grade, thus demonstrating recanalization of the affected vessel (with 1 case improving to a Grade 1 injury and 1 case improving to a Grade 3 injury, and neither patient presenting with adverse clinical symptoms as a result of the recanalization).

With regard to treatment for Grade 3 BCAIs, 33 patients received ASA alone; 1 patient received ASA and clopidogrel, then transitioned to Coumadin; 1 patient received ASA and clopidogrel; and 1 patient received no treatment. The 8 patients who received endovascular treatment continued treatment with ASA, and in those patients who underwent stenting, treatment with clopidogrel was scheduled for 6 months after the procedure. As depicted in Table 1, all of the patients in the Grade 4 BCAI follow-up

TABLE 3. Progression of injury of Grade 3 BCAls at 7-10 days and at final imaging

\begin{tabular}{ccc}
\hline \multirow{2}{*}{ Status } & \multicolumn{2}{c}{ Imaging Status of Injury } \\
\cline { 2 - 3 } & At 7-10 days & At Final Imaging (7-960 days) \\
\hline Resolved & $2 \%(1)$ & $11 \%(6)$ \\
\hline Improved & $11 \%(6)$ & $11 \%(6)$ \\
\hline Stable & $51 \%(27)$ & $53 \%(28)$ \\
\hline Worsened & $36 \%(19)$ & $25 \%(13)$ \\
\hline
\end{tabular}


group were treated with ASA. In the isolated case that was associated with cerebral infarction, the ASA was transitioned to Coumadin.

There were 16 patients with Grade 3 BCAI who underwent additional neuroimaging for evaluation of trauma or symptoms of infarction. Seven of these patients had a Grade 3 BCAI with a pseudoaneurysm-associated vessel stenosis greater than $50 \%$, and 2 of those patients were found to have cerebral infarction. Nine patients had a Grade 3 BCAI with a pseudoaneurysm-associated vessel stenosis of less than $50 \%$, and 1 of those patients was found to have cerebral infarction. Thus, of the 16 patients who underwent posttraumatic imaging for symptomatology or further evaluation, there were 3 cases of identified cerebral infarction ( $7 \%$ of the Grade 3 BCAI follow-up group) that appeared to be related to the BCAI. There was 1 case of cerebral infarction identified in the Grade 4 BCAI followup group. The first identified case of stroke in the Grade 3 BCAI group occurred in 2005 in a 25-year-old man involved in an MVC. He was found to have a right occipital condyle fracture, multiple facial fractures, and a right-sided Grade 3 BCAI between C-1 and the skull base. He did not have any neurological symptoms, and his admission head CT scan did not demonstrate any hypodensities to suggest infarction. He was taken for angiography later that day to allow for additional characterization of the vascular lesion. No endovascular intervention was performed, and he was started on ASA and clopidogrel. He underwent routine head CT scanning the next day, which revealed several asymptomatic, subcentimeter subcortical right frontal and occipital-parietal hypodensities. Although it was arguable whether these infarctions were the result of the procedure, no subsequent MRI was performed, and the patient was discharged 1 week later to his home in stable condition. Follow-up imaging demonstrated stability of his vascular lesion. The second case occurred in 2011 in a 59-year-old woman involved in an MVC who sustained stable fractures of C-2 and C-7 that did not require surgery and a rightsided Grade 3 BCAI between C-1 and the skull base. Treatment with ASA was initiated soon after admission, and on Day 2 of hospitalization, the patient underwent brain MRI to determine the cause of progressive right-arm weakness, which was noted to have developed soon after her admission. The MR image demonstrated an isolated, subcentimeter, right frontal subcortical infarction, which was not believed to correlate with her complaints. The patient's arm weakness resolved shortly thereafter. She was followed up for 1 year and underwent CTA on 5 subsequent occasions, and although the lesion did increase slightly in size compared to the first follow-up image, it was found to be stable on all subsequent imaging. The third case of cerebral infarction occurred in 2012 in a 36-year-old man involved in an all-terrain vehicle accident who presented with a significant left hemiparesis. He was found to have an isolated right-sided Grade 3 BCAI near the skull base, and admission head CT scanning demonstrated profound hypodensity within the right middle cerebral artery distribution. No other significant traumatic findings were identified, and the patient began treatment with ASA and was monitored in the intensive care unit. His neurological exam remained stable, and he was discharged to inpatient rehabilitation. The patient was followed up for 5 months and underwent CTA 3 additional times, which demonstrated improvement in the size of the pseudoaneurysm but persistence of the lesion overall. The Grade 4 BCAI occurred in 2005 in a 24-year-old man who was involved in an MVC and sustained extensive skull base fractures and a right-sided carotid artery occlusion that extended from C-3 to the skull base. His admission head CT scan demonstrated subtle right frontal hypodensity, and the patient began treatment with ASA. Brain MRI was performed on hospital Day 2, and this confirmed a moderately sized right frontal cerebral infarction. No endovascular interventions were performed; however, the lesion was further characterized and confirmed with angiography later that day, and the patient was transitioned subsequently to Coumadin. There were no obvious neurological sequelae as a result of this infarction, and the patient was discharged in stable condition. He was followed up closely for 2 months and underwent CTA 3 additional times during the follow-up period, all of which demonstrated a stable occlusion of the right carotid vessel.

\section{Discussion}

Since the 1990s, BCVI research has led to an increase in screening protocols and treatment strategies for this type of injury. Disagreement persists regarding the appropriate screening methods, treatment, and follow-up examinations of these traumatic vascular injuries. Common parameters for BCVI screening include cases of symptomatic ischemic injuries, asymptomatic Le Fort II/III fractures, cervical spine fractures, Horner's syndrome, foramen lacerum fractures, neck soft-tissue injury, or unexplained ischemic events. ${ }^{4,12}$ There have also been case reports of BCVI arising from more benign torsion injuries, such as those reported from common activities such as yoga. ${ }^{22-24}$

At our institution, trauma patients meeting the criteria for BCVI screening undergo CTA. Formerly considered the gold standard, DSA has been supplanted by CTA as the standard in our center because of the compelling evidence on the topic. ${ }^{2,6,9,15,21,22}$ Biffl et al. ${ }^{5}$ evaluated the progression of BCVI in a sequential DSA study examining both vertebral and carotid artery injuries together as a cohort. In this study, DSA was performed immediately upon admission and then again 7-10 days after admission. The authors found that only $1 \%$ of the Grade 3 BCVIs resolved, whereas the remainder were unchanged, and that $9 \%$ of the Grade 4 BCVIs resolved, whereas $82 \%$ remained unchanged. Because this study combined both carotid and vertebral artery injuries for evaluation, it is difficult to make exact correlations to our current study examining high-grade BCAIs. In addition, we defined improvement as a decrease in pseudoaneurysm size, whereas Biffl et al. did not take this into consideration. Our study demonstrated somewhat similar findings, however, in that the majority $(53 \%)$ of Grade 3 BCAIs remained unchanged at final imaging; however, $22 \%$ of injured arteries did exhibit either improvement or, to a lesser extent, complete resolution, whereas $25 \%$ demonstrated worsening of the pseudoaneurysm. Likewise, as noticed in our previous investigation of Grade 1 and 2 BCAIs, ${ }^{25}$ the majority of low-grade BCAIs that demonstrated a radiographic worsening to a Grade 3 
did not resolve or subsequently improve to a lesser grade but stabilized at this higher grade, correlating with this current study, which shows that at final follow-up examination, $89 \%$ of Grade 3 injuries persisted to variable degrees.

In general, the greatest concern regarding BCVIs, including the high-grade BCAIs, is the risk of cerebral infarction; however, reports have suggested that the risk of ischemic stroke after BCVI may be quite low. Griessenauer et al. ${ }^{12}$ examined the outcomes of 112 patients with BCVI. They included both blunt carotid and vertebral artery injuries of various grades and identified only one case of ischemic stroke caused by BCVI. From the available literature, however, it is difficult to determine the true rate of BCVI-related ischemic strokes, which has been reported to be as low as $0.05 \%$ and as high as $50 \% .^{10,12,14,16}$ In this current study examining high-grade BCAI, we identified 3 patients $(7 \%)$ who presented with a Grade 3 BCAI-related stroke and 1 patient who presented with a Grade 4 BCAIrelated stroke. These higher-grade BCAIs have thus posed the greatest risk of cerebral infarction in our PCVAIS study. ${ }^{25-27}$ An earlier study from our institution by Eastman et al. ${ }^{10}$ examined 26 patients with either a BCAI and/ or a BVAI ( 3 of those patients had a Grade 3 or 4 BCAI) and found a stroke rate of $3.8 \%(1 / 26)$. The cerebral infarction in this study was identified in a patient with a Grade 3 injury. Griessenauer et al. ${ }^{12}$ concluded that most ischemic strokes occur before initial screening with CTA and before treatment with an antiplatelet medication, indicating that follow-up imaging may not aid in preventing most ischemic strokes. We have noticed similar findings thus far in our BCVI analysis, with the majority if not all of the identified strokes presenting soon after admission and frequently before the administration of any medication. ${ }^{25-27}$ Likewise, Mayberry et al. ${ }^{16}$ retrospectively examined data from two major trauma centers and noted that all strokes due to BCAI occurred within 12 hours of admission. It is noteworthy that 8 of our patients were lost to follow-up after hospital discharge. However, these patients were evaluated by both our trauma and neurosurgery teams for an average of 13 days (range 1-60 days). All patients in this group were identified as being in stable condition at their discharge, and all individuals had undergone posttraumatic head CT scanning in addition to CTA for diagnosis of their BCAI. There were no clinical or radiographic cerebral infarctions identified in this group, suggesting that the peritraumatic stroke rate may be closer to $5 \%$. If it is argued that the scattered asymptomatic subcortical infarctions in the first presented infarction case were the unintended iatrogenic result of a postprocedural angiographic complication, the stroke rate may further decrease to 3.5\%. Despite this, high-grade BCAIs represent the greatest risk of stroke when compared with our earlier investigations on lowgrade BCAIs and all grades of BVAIs. ${ }^{25-27}$

The progression of injury does not seem to be affected by any specific medical treatment(s). Cothren et al. ${ }^{8}$ evaluated 422 patients with BCVI, 260 of whom had either a Grade 3 or 4 vertebral or carotid artery injury. The majority of patients in their cohort (49\%) were treated with heparin, 23\% were treated with either ASA or a combination of ASA and clopidogrel, and 28\% were not treated. The authors concluded that there was no reliable way to alter the progression of injury with or without medication. Although our earlier investigations on low-grade BCAI and BVAI noted similar results, ${ }^{25,27}$ our current study of high-grade BCAIs, similar to our investigation of highgrade BVAIs, lacked the variety of treatment required to suggest variability in injury progression based solely on the presence or absence of treatment, given that nearly the entire cohort received ASA. Although those individuals with Grade 3 BCAIs receiving endovascular intervention were all identified as having stable findings on follow-up imaging, previous studies have reported varying efficacy and safety for the endovascular treatment of pseudoaneurysms. ${ }^{1,4,7,11,13,19} \mathrm{Li}$ et al. ${ }^{14}$ performed a national trauma bank review concluding that there was no difference in the functional outcome between patients treated with endovascular procedures and those treated with medicine alone.

The major limitation of this study was its retrospective design. A prospective study is needed to implement various treatment strategies to verify appropriate followup protocols. Although some studies have suggested that CTA overestimates vascular injury in these blunt trauma cases, ${ }^{12,18,20,29}$ we used it in the majority of cases because of its associated comparative ease, accessibility, and safety compared with DSA, especially in critically ill patients who have sustained multisystem trauma. Use of CTA may have thus produced a falsely elevated rate of vascular injury at our center. Because these injuries reflect percentages of vessel diameter disruption, a falsely elevated rate of improvement on follow-up imaging may also be reflected in this study compared with previous studies using DSA, $, 5,17$ especially if there was a potential overestimation of initial injury. Another limitation of this study was the lack of treatment variety in the high-grade BCAI group that would be necessary to comment on injury progression based on the presence or absence of treatment.

Because of the dynamic nature of our study population, and at times following multiple vascular injuries simultaneously, we experienced a large variability in our followup days, which did limit our ability to conclusively state timing related to the healing of these higher-grade carotid artery injuries. However, that does not diminish the fact that most of these injuries proved to be stable or had improved at final follow-up evaluation. Another limitation of this retrospective investigation is that we cannot be entirely sure that some clinically silent strokes were not missed; however, we can offer that all individuals included in this study did undergo follow-up evaluations, and thus, we are certain that all clinically apparent strokes were both identified and recorded. Although these data accumulated over the past 10 years at our institution have yielded valuable information, the data involving high-grade carotid artery injuries alone did not afford much ability for statistical analysis; however, once all of the data across all of the cervical vascular injuries have been further analyzed, we look forward to offering a well-powered analysis across all grades of blunt vertebral and carotid injuries, specifically as in relation to the risk of stroke in the setting of treatment, timing, vessel, and grade.

\section{Conclusions}

The posttraumatic cerebral infarction rate for high- 
grade BCAI may be overestimated; however, results of this study suggest that the Grade 3 and 4 BCAIs carry the highest stroke risk of the BCVIs; those infarctions were identified either upon or soon after hospital admission. Despite a $40 \%$ recanalization rate in the Grade 4 BCAI group and an $89 \%$ rate of persistent pseudoaneurysm in the Grade 3 BCAI group, follow-up imaging showed progressive worsening without radiographic improvement in only a small number of patients, and these findings alone did not correlate with adverse clinical outcome. Followup protocols may require amending; however, further prospective studies are needed to make conclusive changes in management.

\section{References}

1. Bendszus M, Koltzenburg M, Burger R, Warmuth-Metz M, Hofmann E, Solymosi L: Silent embolism in diagnostic cerebral angiography and neurointerventional procedures: a prospective study. Lancet 354:1594-1597, 1999

2. Biffl WL, Cothren CC, Moore EE, Kozar R, Cocanour C, Davis JW, et al: Western Trauma Association critical decisions in trauma: screening for and treatment of blunt cerebrovascular injuries. J Trauma 67:1150-1153, 2009

3. Biffl WL, Moore EE, Offner PJ, Brega KE, Franciose RJ, Burch JM: Blunt carotid arterial injuries: implications of a new grading scale. J Trauma 47:845-853, 1999

4. Biffl WL, Moore EE, Offner PJ, Burch JM: Blunt carotid and vertebral arterial injuries. World J Surg 25:1036-1043, 2001

5. Biffl WL, Ray CE Jr, Moore EE, Franciose RJ, Aly S, Heyrosa MG, et al: Treatment-related outcomes from blunt cerebrovascular injuries: importance of routine follow-up arteriography. Ann Surg 235:699-707, 2002

6. Biffl WL, Ray CE Jr, Moore EE, Mestek M, Johnson JL, Burch JM: Noninvasive diagnosis of blunt cerebrovascular injuries: a preliminary report. J Trauma 53:850-856, 2002

7. Cafasso D, Meadows JM, Wolfe SQ, Katras T, Kellicut DC, Golarz SR: Endovascular treatment of bilateral carotid artery pseudoaneurysms after blunt carotid injury. Ann Vasc Surg 28:e11-e16, 2014

8. Cothren CC, Biffl WL, Moore EE, Kashuk JL, Johnson JL: Treatment for blunt cerebrovascular injuries: equivalence of anticoagulation and antiplatelet agents. Arch Surg 144:685690, 2009

9. Eastman AL, Chason DP, Perez CL, McAnulty AN, Minei JP: Computed tomographic angiography for the diagnosis of blunt cervical vascular injury: is it ready for primetime? J Trauma 60:925-929, 2006

10. Eastman AL, Muraliraj V, Sperry JL, Minei JP: CTA-based screening reduces time to diagnosis and stroke rate in blunt cervical vascular injury. J Trauma 67:551-556, 2009

11. Edwards NM, Fabian TC, Claridge JA, Timmons SD, Fischer PE, Croce MA: Antithrombotic therapy and endovascular stents are effective treatment for blunt carotid injuries: results from longterm followup. J Am Coll Surg 204:1007-1015, 2007

12. Griessenauer CJ, Fleming JB, Richards BF, Cava LP, Curé JK, Younan DS, et al: Timing and mechanism of ischemic stroke due to extracranial blunt traumatic cerebrovascular injury. J Neurosurg 118:397-404, 2013

13. Kansagra AP, Cooke DL, English JD, Sincic RM, Amans MR, Dowd CF, et al: Current trends in endovascular management of traumatic cerebrovascular injury. J Neurointerv Surg 6:47-50, 2013

14. Li W, D'Ayala M, Hirshberg A, Briggs W, Wise L, Tortolani A: Comparison of conservative and operative treatment for blunt carotid injuries: analysis of the National Trauma Data Bank. J Vasc Surg 51:593-599.e2, 2010

15. Liang T, Plaa N, Tashakkor AY, Nicolaou S: Imaging of blunt cerebrovascular injuries. Semin Roentgenol 47:306-319, 2012
16. Mayberry JC, Brown CV, Mullins RJ, Velmahos GC: Blunt carotid artery injury: the futility of aggressive screening and diagnosis. Arch Surg 139:609-613, 2004

17. Miller PR, Fabian TC, Bee TK, Timmons S, Chamsuddin A, Finkle R, et al: Blunt cerebrovascular injuries: diagnosis and treatment. J Trauma 51:279-286, 2001

18. Miller PR, Fabian TC, Croce MA, Cagiannos C, Williams JS, Vang M, et al: Prospective screening for blunt cerebrovascular injuries: analysis of diagnostic modalities and outcomes. Ann Surg 236:386-395, 2002

19. Moulakakis KG, Mylonas S, Avgerinos E, Kotsis T, Liapis CD: An update of the role of endovascular repair in blunt carotid artery trauma. Eur J Vasc Endovasc Surg 40:312-319, 2010

20. Mutze S, Rademacher G, Matthes G, Hosten N, Stengel D: Blunt cerebrovascular injury in patients with blunt multiple trauma: diagnostic accuracy of duplex Doppler US and early CT angiography. Radiology 237:884-892, 2005

21. Provenzale JM, Morgenlander JC, Gress D: Spontaneous vertebral dissection: clinical, conventional angiographic, CT, and MR findings. J Comput Assist Tomogr 20:185-193, 1996

22. Roberts DJ, Chaubey VP, Zygun DA, Lorenzetti D, Faris PD, Ball CG, et al: Diagnostic accuracy of computed tomographic angiography for blunt cerebrovascular injury detection in trauma patients: a systematic review and meta-analysis. Ann Surg 257:621-632, 2013

23. Russell WR: Yoga and vertebral arteries. Br Med J 1:685, 1972

24. Schievink WI: Spontaneous dissection of the carotid and vertebral arteries. N Engl J Med 344:898-906, 2001

25. Scott WW, Sharp S, Figueroa SA, Eastman AL, Hatchette CV, Madden CJ, et al: Clinical and radiographic outcomes following traumatic Grade 1 and 2 carotid artery injuries: a 10-year retrospective analysis from a Level 1 trauma center. J Neurosurg [in press], 2014

26. Scott WW, Sharp S, Figueroa SA, Eastman AL, Hatchette $\mathrm{CV}$, Madden CJ, et al: Clinical and radiographic outcomes following traumatic Grade 3 and 4 vertebral artery injuries: a 10 -year retrospective analysis from a Level 1 trauma center. The Parkland Carotid and Vertebral Artery Injury Survey. J Neurosurg [epub ahead of print October 24, 2014. DOI: 10.3171/2014.9.JNS1461], 2014

27. Scott WW, Sharp S, Figueroa SA, Madden CJ, Rickert KL: Clinical and radiographic outcomes following traumatic Grade 1 and 2 vertebral artery injuries: a 10-year retrospective analysis from a Level 1 trauma center. J Neurosurg 121:450-456, 2014

28. Wahl WL, Brandt MM, Thompson BG, Taheri PA, Greenfield LJ: Antiplatelet therapy: an alternative to heparin for blunt carotid injury. J Trauma 52:896-901, 2002

29. Wang AC, Charters MA, Thawani JP, Than KD, Sullivan SE, Graziano GP: Evaluating the use and utility of noninvasive angiography in diagnosing traumatic blunt cerebrovascular injury. J Trauma Acute Care Surg 72:1601-1610, 2012

\section{Author Contributions}

Conception and design: Scott, Rickert. Acquisition of data: Scott, Sharp, Madden, Rickert. Analysis and interpretation of data: Scott, Sharp, Figueroa, Eastman, Rickert. Drafting the article: Scott, Sharp. Critically revising the article: Scott, Figueroa, Eastman, Hatchette, Madden, Rickert. Reviewed submitted version of manuscript: all authors. Approved the final version of the manuscript on behalf of all authors: Scott. Administrative/technical/material support: Madden. Study supervision: Rickert.

\section{Correspondence}

William W. Scott, Department of Neurosurgery, University of Texas Southwestern Medical Center, 5323 Harry Hines Blvd., Dallas, TX 75390-8855. email: william_w_scott@hotmail.com. 